\title{
A GENERALIZATION OF THE COMPLEX INVERSION FORMULA FOR THE LAPLACE TRANSFORMATION
}

\author{
P. G. ROONEY
}

1. Introduction. The purpose of this paper is to show that if

I

$$
f(s)=\int_{0}^{\infty} e^{-s t} \phi(t) d t,
$$

then, under the same conditions as those for the complex inversion formula ${ }^{1}$ (see [4, chap. II, §7]),

II

$$
\phi(t)=(2 \pi i \Gamma(1-\beta))^{-1} P \int_{\gamma-i \infty}^{\gamma+i \infty}(s t)^{-\beta} F_{1}(1 ; 1-\beta ; s t) f(s) d s,
$$

if $\operatorname{Re} \beta>0, \beta \neq 1,2, \cdots$. We shall also give conditions for the use of this formula when $\operatorname{Re} \beta \leqq 0$.

The inversion formula II may be considered as a generalization of the complex inversion formula, since for $\beta=0$, it reduces to that formula.

The formula also holds, in a sense, when $\beta=1,2, \cdots$; for, since

$$
(\Gamma(1-\beta))^{-1}{ }_{1} F_{1}(1 ; 1-\beta ; s t)=\sum_{r=0}^{\infty}(\Gamma(r+1-\beta))^{-1}(s t)^{r},
$$

it seems natural to consider this expression, for $\beta=n$, equal to $(s t)^{n} e^{s t}$ thus reproducing the complex inversion formula.

Formula II can be derived under considerably more rigid conditions than those for the complex inversion formula, by means of integrals of fractional order. We shall not do this here.

2. $\operatorname{Re} \beta$ positive.

Theorem 1. If

(1) $e^{-\gamma t} \phi(t) \in L(0, \infty)$, $\gamma>0$,

(2) $f(s)=\int_{0}^{\infty} e^{-s t} \phi(t) d t$ $\operatorname{Re} s \geqq \gamma$,

(3) $\phi(u)$ is of bounded variation in a neighbourhood of $u=t>0$,

(4) $\operatorname{Re} \beta>0$, $\beta \neq 1,2, \cdots$,

Received by the editors June 24, 1953 and, in revised form, October 30, 1953.

1 The referee has pointed out to me that formula II is a special case of equation (5.2) in A. Erdélyi, Rend. Sem. Mat. Univ. e Polit. di Torino vol. 10 (1950-51) pp. 217-234. However, the methods of proof are totally different. 
then

$$
\begin{aligned}
(2 \pi i \Gamma(1-\beta))^{-1} P \int_{\gamma-i \infty}^{\gamma+i \infty}(s t)^{-\beta} F_{1}(1 ; 1-\beta ; s t) f(s) d s & \\
& =\frac{1}{2}(\phi(t+)+\phi(t-)) .
\end{aligned}
$$

Proof. By $[3$, p. 87 $],{ }_{1} F_{1}(1 ; 1-\beta ; s t)=e^{s t}{ }_{1} F_{1}(-\beta ; 1-\beta ;-s t)$. Now

$$
\begin{aligned}
\frac{d}{d z}\left(z^{-\beta} F_{1}(-\beta ; 1-\beta ;-z)\right) & =\frac{d}{d z}\left(-\beta \sum_{n=0}^{\infty}(-1)^{n}((n-\beta)(n !))^{-1} z^{n-\beta}\right) \\
& =-\beta z^{-\beta-1} e^{-z} .
\end{aligned}
$$

Thus integrating from $z$ to infinity, and using the fact that

$$
\lim _{x \rightarrow \infty} F_{1} F_{1}(-\beta ; 1-\beta ;-x)=\Gamma(1-\beta) \quad \text { (see }[1 ; \text { p. 265]), }
$$

we have, for $\operatorname{Re} z>0$,

$$
z^{-\beta} F_{1}(-\beta ; 1-\beta ;-z)=\Gamma(1-\beta)+\beta \int_{z}^{\infty} u^{-\beta-1} e^{-n} d u .
$$

Thus when $s$ and $t$ are real and positive we have

$(\Gamma(1-\beta))^{-1}(s t)^{-\beta_{1}} F_{1}(-\beta ; 1-\beta ;-s t)$

$$
\begin{aligned}
& =1-(\Gamma(-\beta))^{-1} \int_{s t}^{\infty} u^{-\beta-1} e^{-n} d u \\
& =1-(\Gamma(-\beta))^{-1} t^{-\beta} \int_{s}^{\infty} x^{-\beta-1} e^{-t x} d x
\end{aligned}
$$

and, by analytic continuation, this formula must hold for all $t>0$ and all $s$, such that $\operatorname{Re} s>0$. Now

$$
\int_{s}^{\infty} x^{-\beta-1} e^{-t x} d x=\int_{0}^{\infty} e^{-s y} \psi(y) d y, \quad \operatorname{Re} s>0,
$$

where $\psi(y)=0,0 \leqq y<t$, and equals $(\Gamma(\beta+1))^{-1}(y-t)^{\beta} y^{-1}, y \geqq t$. Hence

$$
(\Gamma(1-\beta))^{-1}(s t)^{-\beta} F_{1}(-\beta ; 1-\beta ; s t) f(s)=f(s)-(\Gamma(-\beta))^{-1} t^{-\beta} g(s),
$$

where $g(s)=\int_{0}^{\infty} e^{-s y}[\phi * \psi](y) d y$, Re $s \geqq \gamma$, and $[p * q](y)=[q * p](y)$ $=\int_{0}^{y} p(y-x) q(x) d x$. Further, 


$$
[\psi * \phi](y)=\left\{\begin{array}{lr}
0, & 0 \leqq y<t, \\
(\Gamma(\beta+1))^{-1} \int_{0}^{y-t}((y-t)-x)^{\beta}(y-x)^{-1} \phi(x) d x, & y \geqq t,
\end{array}\right.
$$

so that $[\psi * \phi](t-)=0$, and

$$
\begin{array}{rlr}
|[\psi * \phi](y)| & =0 & (0<y<t) \\
& \leqq|\Gamma(1+\beta)|^{-1}(y-t)^{\operatorname{Re} \beta} t^{-1} \int_{0}^{\delta}|\phi(x)| d x, & 0<y-t<\delta,
\end{array}
$$

and hence $[\psi * \phi](t+)=0$, so that $[\psi * \phi](y)$ satisfies the conditions of [4, chap. II, Theorem 7.4]. Thus, by [4, chap. II, Theorems 7.3 and 7.4$]$,

$$
\begin{aligned}
(2 \pi i \Gamma(1-\beta))^{-1} P \int_{\gamma-i \infty}^{\gamma+i \infty}(s t)^{-\beta} F_{1}(1 ; 1-\beta ; s t) f(s) d s \\
=(2 \pi i)^{-1} P \int_{\gamma-i \infty}^{\gamma+i \infty} e^{s t} f(s) d s+(2 \pi i \Gamma(-\beta))^{-1} t^{-\beta} P \int_{\gamma-i \infty}^{\gamma+i \infty} e^{s t} g(s) d s \\
=\frac{1}{2}(\phi(t+)+\phi(t-)) \\
\quad+\frac{1}{2}(\Gamma(-\beta))^{-1} t^{-\beta}([\psi * \phi](t+)+[\psi * \phi](t-)) \\
=\frac{1}{2}(\phi(t+)+\phi(t-)) .
\end{aligned}
$$

\section{3. $\operatorname{Re} \beta$ negative.}

THEOREM 2. If $\phi(t)$ satisfies (1), (2), and (3) of Theorem 1, and if

(4) $\operatorname{Re} \beta \leqq 0$,

(5) $\alpha$ exists, $\operatorname{Re} \alpha>-\operatorname{Re} \beta$, such that

$$
\theta(t)=(\Gamma(n-\alpha))^{-1} \int_{0}^{t}(t-x)^{\alpha-1} \phi(x) d x
$$

is $C^{(n-1)}(t \geqq 0)$, where $n=[\operatorname{Re} \alpha]+1, \theta^{(n-1)}(t)$ is absolutely continuous for $t \geqq 0, \theta^{(r)}(0)=0, r=0, \cdots, n-1, \theta^{(n-1)}(t)=o\left(e^{\gamma t}\right), t \rightarrow \infty$, then

$$
\begin{aligned}
(2 \pi i \Gamma(1-\beta))^{-1} P \int_{\gamma-i \infty}^{\gamma+i \infty}(s t)^{-\beta} F_{1}(1 ; 1-\beta ; s t) f(s) d s & \\
& =\frac{1}{2}(\phi(t+)+\phi(t-)) .
\end{aligned}
$$


Proof. Let $\theta^{(n-1)}(t)=\int_{0}^{t} \psi(x) d x$. Then plainly

$$
\phi(t)=(\Gamma(\alpha))^{-1} \int_{0}^{t}(t-x)^{\alpha-1} \psi(x) d x,
$$

and $F(s)=s^{-\alpha} g(s), s \geqq \gamma$ where $g(s)=\int_{0}^{-\infty} e^{-s} \psi(t) d t$, and $\int_{0}^{\rightarrow \infty} p(t) d t$ $\equiv \lim _{R \rightarrow \infty} \int_{0}^{R} p(t) d t$.

Now if $\beta=0$, the theorem follows from [4, chap. II, Theorem 7.3]. Suppose, then, $-1<\operatorname{Re} \beta \leqq 0, \beta \neq 0$. Now

$$
\begin{aligned}
& (\Gamma(1-\beta))^{-1}(s t)^{-\beta}{ }_{1} F_{1}(1 ; 1-\beta ; s t) \\
& \quad=\left(\Gamma\left(1-\beta^{\prime}\right)\right)^{-1}(s t)^{-\beta^{\prime}}+\left(\Gamma\left(1-\beta^{\prime}\right)\right)^{-1}(s t)^{-\beta^{\prime}}{ }_{1} F_{1}\left(1 ; 1-\beta^{\prime} ; s t\right),
\end{aligned}
$$

where $\beta^{\prime}=\beta+1$, as can be verified by writing the series for each side of the above equation. Thus, by Theorem 1 ,

$$
\begin{aligned}
& (2 \pi i \Gamma(1-\beta))^{-1} P \int_{\gamma-i \infty}^{\gamma+i \infty}(s t)^{-\beta} F_{1}(1 ; 1-\beta ; s t) f(s) d s \\
& \quad=\left(2 \pi i \Gamma\left(1-\beta^{\prime}\right)\right)^{-1} P \int_{\gamma-i \infty}^{\gamma+i \infty}(s t)^{-(\beta+1)} f(s) d s+\frac{1}{2}(\phi(t+)+\phi(t-)),
\end{aligned}
$$

since $0<\operatorname{Re} \beta^{\prime} \leqq 1$.

But $s^{-(\beta+1)} f(s)=s^{-(\alpha+\beta+1)} g(s)$. Now $s^{-1} g(s)=\int_{0}^{\rightarrow \infty} e^{-8 y} \psi_{1}(y) d y$, Re $s$ $\geqq \gamma$, where $\psi_{1}(y)=\int_{0}^{\nu} \psi(x) d x=\theta^{(n-1)}(y)$ a.e. Hence, by (5),

$$
s^{-1} g(s)=\int_{0}^{\infty} e^{-s y \theta^{(n-1)}}(y) d y=\int_{0}^{\infty} e^{-s y} \psi_{1}(y) d y .
$$

Thus, by [4, chap. II, §8], $s^{-(\alpha+\beta+1)} g(s)=\int_{0}^{\infty} e^{-s y} \psi_{2}(y) d y, \operatorname{Re} s \geqq \gamma$, where

$$
\begin{aligned}
\psi_{2}(y) & =(\Gamma(\alpha+\beta))^{-1} \int_{0}^{y}(y-x)^{\alpha+\beta-1} \psi_{1}(x) d x \\
& =(\Gamma(\alpha+\beta+1))^{-1} \int_{0}^{y}(y-x)^{\alpha+\beta} \psi(x) d x .
\end{aligned}
$$

Further

$$
\begin{aligned}
& \left|\psi_{2}(y)\right| \leqq|\Gamma(\alpha+\beta+1)|^{-1} y^{\mathrm{Re}(\alpha+\beta)} \int_{0}^{y}|\psi(x)| d x, \quad y>0, \\
& \leqq|\Gamma(\alpha+\beta+1)|^{-1} y^{\operatorname{Re}(\alpha+\beta)} \int_{0}^{\delta}|\psi(x)| d x, \quad 0<y<\delta
\end{aligned}
$$

so that $\psi_{2}(0+)=0$, and $\psi_{2}(y)$ satisfies the conditions of [4, chap. II, 
Theorem 7.4]. Hence, $(2 \pi i)^{-1} P \int_{\gamma-i \infty}^{\gamma+i \infty} s^{-(\beta+1)} f(s) d s=\psi_{2}(0+) / 2=0$.

Next suppose $-(k+1)<\operatorname{Re} \beta \leqq-k$, and suppose the theorem is true for $-k<\operatorname{Re} \beta \leqq-(k-1)$. Then, as before,

$$
\begin{aligned}
& (\Gamma(1-\beta))^{-1}(s t)^{-\beta_{1} F_{1}(1 ; 1-\beta ; s t)} \\
& =\left(\Gamma\left(1-\beta^{\prime}\right)\right)^{-1}(s t)^{-\beta^{\prime}}+\left(\Gamma\left(1-\beta^{\prime}\right)\right)^{-1}(s t)^{-\beta^{\prime}}{ }_{1} F_{1}\left(1 ; 1-\beta^{\prime} ; s t\right),
\end{aligned}
$$

where $\beta^{\prime}=\beta+1$, and thus since $-k<\operatorname{Re} \beta^{\prime} \leqq-(k-1)$, we have

$$
\begin{aligned}
& (2 \pi i \Gamma(1-\beta))^{-1} P \int_{\gamma-i \infty}^{\gamma+i \infty}(s t)^{-\beta} F_{1}(1 ; 1-\beta ; s t) f(s) d s \\
& \quad=\left(2 \pi i \Gamma\left(1-\beta^{\prime}\right)\right)^{-1} P \int_{\gamma-i \infty}^{\gamma+i \infty}(s t)^{-(\beta+1)} f(s) d s+\frac{1}{2}(\phi(t+)+\phi(t-)),
\end{aligned}
$$

and the integral on the right-hand side of this equation is zero in exactly the same manner as previously. Hence by induction the theorem is true for all $\beta, \operatorname{Re} \beta \leqq 0$.

We can improve our results slightly if $\beta$ is real as is shown by the following theorem.

Theorem 3. If $\phi(t)$ satisfies (1), (2), and (3) of Theorem 1, and if

(4) $\beta \leqq 0$,

(5) $\theta(t)=(\Gamma(n+\beta))^{-1} \int_{0}^{t}(t-x)^{n+\beta-1} \phi(x) d x \in C^{(n-1)}(t \geqq 0)$, where $n$ $=[-\beta]+1, \theta^{(n-1)}(t)$ is absolutely continuous in $t \geqq 0, \theta^{(n-1)}(t)=o\left(e^{\gamma t}\right)$ as $t \rightarrow \infty, \theta^{(r)}(0)=0, r=0, \cdots, n-1$, then

$$
\begin{aligned}
(2 \pi i \Gamma(1-\beta))^{-1} P \int_{\gamma-i \infty}^{\gamma+i \infty}(s t)^{-\beta} F_{1}(1 ; 1-\beta ; s t) f(s) d s & \\
& =\frac{1}{2}(\phi(t+)+\phi(t-)) .
\end{aligned}
$$

Proof. Let $\theta^{(n-1)}(t)=\int_{0}^{t} \psi(x) d x$. Then plainly

$$
\phi(t)=(\Gamma(1-\beta))^{-1} \int_{0}^{t}(t-x)^{-\beta-1} \psi(x) d x,
$$

and $f(s)=s^{-\beta} g(s)$, where $g(s)=\int_{0}^{\rightarrow \infty} e^{-s t} \psi(t) d t$. Then, as in the previous theorem, we have

$$
\begin{aligned}
& (2 \pi i \Gamma(1-\beta))^{-1} P \int_{\gamma-i \infty}^{\gamma+i \infty}(s t)^{-\beta_{1}} F_{1}(1 ; 1-\beta ; s t) f(s) d s \\
& \quad=\left(2 \pi i \Gamma\left(1-\beta^{\prime}\right)\right)^{-1} P \int_{\gamma-i \infty}^{\gamma+i \infty}(s t)^{-(\beta+1)} f(s) d s+\frac{1}{2}(\phi(t+)+\phi(t-)),
\end{aligned}
$$


where $\beta^{\prime}=\beta+1$, and the theorem will be proved if we show that

$$
(2 \pi i)^{-1} P \int_{\gamma-i \infty}^{\gamma+i \infty} s^{-(\beta+1)} f(s) d s=0 .
$$

But $s^{-(\beta+1)} f(s)=s^{-1} g(s)$. Hence, by [2, Kap. 6, §5, Satz 3],

$$
\begin{aligned}
(2 \pi i)^{-1} P \int_{\gamma-i \infty}^{\gamma+i \infty} s^{-(\beta+1)} f(s) d s & =(2 \pi i)^{-1} P \int_{\gamma-i \infty}^{\gamma+i \infty} s^{-1} g(s) d s \\
& =\left.\frac{1}{2} \int_{0}^{t} \psi(y) d y\right|_{t=0+}=0 .
\end{aligned}
$$

4. Some special cases. By giving particular values to $\beta$, and using well known relations involving Kummer functions, we can deduce various new inversion formulas. We give two of these here.

Thus if $\beta=-1 / 2$, we have

$$
\begin{aligned}
& \frac{1}{2}(\phi(t+)+\phi(t-)) \\
& \quad=\left(2 \pi i \Gamma\left(\frac{3}{2}\right)\right)^{-1} P \int_{\gamma-i \infty}^{\gamma+i \infty}(s t)^{1 / 2}{ }_{1} F_{1}\left(1 ; \frac{3}{2} ; s t\right) f(s) d s \\
& \quad=\left(2 \pi i \Gamma\left(\frac{3}{2}\right)\right)^{-1} P \int_{\gamma-i \infty}^{\gamma+i \infty} e^{s t}(s t)^{1 / 2} F_{1}\left(\frac{1}{2} ; \frac{3}{2} ;-s t\right) f(s) d s \\
& \quad=(2 \pi i)^{-1} P \int_{\gamma-i \infty}^{\gamma+i \infty} e^{s t} \operatorname{erf}\left((s t)^{1 / 2}\right) f(s) d s \quad \text { [3, page 87] page 96], }
\end{aligned}
$$

this formula holding under the conditions for the complex inversion formula, and that $\theta(t)=\pi^{-1 / 2} \int_{0}^{t}(t-x)^{-1 / 2} \phi(x) d x$ be absolutely continuous, $\theta(t)=o\left(e^{\gamma t}\right)$ as $t \rightarrow \infty$, and $\theta(0+)=0$.

Also, if $\beta=-1$, we have

$$
\begin{aligned}
\frac{1}{2}(\phi(t+)+\phi(t-)) & =(2 \pi i)^{-1} P \int_{\gamma-i \infty}^{\gamma+i \infty}(s t)_{1} F_{1}(1 ; 2 ; s t) f(s) d s \\
& =(\pi i)^{-1} P \int_{\gamma-i \infty}^{\gamma+i \infty} e^{s t / 2} \sinh (s t / 2) f(s) d s
\end{aligned}
$$

by $[3$, pp. 18,19 , and 87$]$. This formula holds under the conditions for the complex inversion formula, and the conditions that $\phi(t)$ be absolutely continuous, $\phi(t)=o\left(e^{\gamma t}\right)$ as $t \rightarrow \infty$, and $\phi(0+)=0$. 


\section{REFERENCES}

1. E. T. Copson, Theory of functions of a complex variable, Oxford, 1935.

2. G. Doetsch, Theorie und Anwendung der Laplace Transformation, Berlin, Springer, 1937.

3. W. Magnus and F. Oberhettinger, Special functions of mathematical physics, New York, Chelsea, 1949.

4. D. V. Widder, The Laplace transform, Princeton, 1941.

University of Alberta

\section{ON FUNDAMENTAL MATRIX SOLUTIONS}

\section{HYMAN J. ZIMMERBERG}

In this note a recent result of Miller and Schiffer $[3, \S 3]$ will be extended to show how a fundamental matrix solution for the differential equations of a system of two point boundary value problems, written in matrix form as

$$
\mathcal{L}[y] \equiv y^{\prime}-A(x) y=0, \quad s[y] \equiv M y(a)+N y(b)=0,
$$

can be represented in terms of the Green's matrix of the system, or in terms of a special generalized Green's matrix in case the system (1) is compatible. For the system (1), $A(x)$ is an $n \times n$ matrix of complex-valued continuous functions of the real variable $x$ on the finite interval $a \leqq x \leqq b, M$ and $N$ are $n \times n$ complex constant matrices such that the rank of the $n \times 2 n$ matrix $\|M N\|$ is $n$, while the vector $y$ will be treated as an $n \times 1$ matrix.

1. In this section we shall assume that the system (1) is incompatible. Then a unique Green's matrix exists and is given by (Bliss $[1, \S 5])$

$$
G(x, t)=\frac{1}{2} Y(x)\left[\frac{|x-t|}{x-t} I+D^{-1} \Delta\right] Y^{-1}(t),
$$

where $Y(x)$ is a fundamental matrix solution of $\mathcal{L}[y]=0, D \equiv M Y(a)$ $+N Y(b), \Delta \equiv M Y(a)-N Y(b)$, and $I$ is the $n \times n$ identity matrix.

THEOREM. If $G(x, t)$ is the ordinary Green's matrix for an incompatible system (1) and $i_{1}, i_{2}, \cdots, i_{2 n}$ denotes a renumbering of the columns of the $n \times 2 n$ matrix $\|M N\|$ such that the $i_{1}, \cdots, i_{n}$ columns are linearly independent vectors, then the corresponding $i_{1}, \cdots, i_{n}$ columns of the $n \times 2 n$ matrix $\|G(x, a) G(x, b)\|$ may be chosen as the columns for a fundamental matrix solution of the differential equations $\mathcal{L}[y]=0$.

Presented, in part, to the Society, October 25, 1952, under the title On the Green's matrix of a differential system; received by the editors August 7, 1953. 